\title{
AV Block First Degree by ECG Finding
}

National Cancer Institute

\section{Source}

National Cancer Institute. AV Block First Degree by ECG Finding. NCI Thesaurus. Code C111088.

An electrocardiographic finding of prolonged PR interval for a specific population. For adults one common threshold is a PR interval greater than 0.20 seconds. Note that other thresholds may be applicable. (CDISC) 